\title{
Prevalencia de anticuerpos contra Chlamydophila abortus en bovinos de crianza extensiva de tres distritos de Ayacucho, Perú
}

\author{
Prevalence of antibodies against Chlamydophila abortus in cattle under extensive \\ system in three districts of Ayacucho, Peru
}

Jean Pierre Loli-Pin ${ }^{1}$, Eglinton Villacaqui-Ayllón ${ }^{1}$, Siever Morales-Cauti, ${ }^{1,2}$

\section{Resumen}

El objetivo del estudio fue determinar la prevalencia de anticuerpos contra Chlamydophila abortus en el ganado bovino de los distritos de Coracora, Chumpi y Pullo de la provincia de Parinacochas, departamento de Ayacucho, Perú. Los animales eran criados de forma extensiva y bajo monta natural. Se tomaron 184 muestras de sangre en bovinos mayores de 4 meses indistintamente del sexo. La detección de anticuerpos contra C. abortus se hizo con un kit comercial de ELISA indirecto. Se obtuvo una prevalencia corregida de $40.34 \pm 7.06 \%$ con diferencia significativa entre distritos $(p<0.05)$, siendo menor en Coracora (40.29 $\pm 8.89 \%$ ) y mayor en Pullo $(56.05 \pm 7.15 \%$, y sin diferencias significativas por efecto del sexo, raza, categoría animal y estado reproductivo. La alta prevalencia encontrada confirma la diseminación del patógeno en la población bovina de los distritos evaluados.

Palabras clave: Chlamydophila, anticuerpos, bovino, ganado, ELISA, Ayacucho

\section{AbSTRaCT}

The aim of this study was to determine the prevalence of antibodies against Chlamydophila abortus in cattle from the districts of Coracora, Chumpi and Pullo of the province of Parinacochas, department of Ayacucho, Peru. The animals were raised extensively and under natural breeding. In total, 184 blood samples were taken from cattle

\footnotetext{
${ }^{1}$ Laboratorio de Microbiología, Carrera de Medicina Veterinaria y Zootecnia, Universidad Cientifica del Sur, Lima, Perú

${ }^{2}$ Email: sieverm@hotmail.com
}

Recibido: 14 de junio de 2020

Aceptado por publicación: 18 de diciembre de 2020

Publicado: 24 de abril de 2021 
over 4 months of age regardless of sex. The detection of antibodies against $C$. abortus was done with a commercial indirect ELISA kit. A corrected prevalence of $40.34 \pm 7.06 \%$ was obtained with a significant difference between districts $(\mathrm{p}<0.05)$, being lower in Coracora (40.29 $\pm 8.89 \%$ ) and higher in Pullo (56.05 $\pm 7.15 \%)$, and without significant differences due to the effect of sex. breed, animal category and reproductive status. The high prevalence confirms the spread of the pathogen in the bovine population of the evaluated districts.

Key words: Chlamydophila, antibodies, bovine, cattle, ELISA, Ayacucho

\section{INTRODUCCIÓN}

La crianza bovina en el Perú es principalmente de doble propósito (carne/leche), teniendo una distribución de $78 \%$ en la sierra, $11 \%$ en la costa y $10 \%$ en la selva. La provincia de Parinacochas en el departamento de Ayacucho se caracteriza por una crianza extensiva de ganado bovino, con una población de 49275 cabezas (Aronés y Castillo, 2003; INEI, 2012; Rosemberg, 2012), la cual se ve afectada por diversas enfermedades reproductivas que ocasionan importantes pérdidas económicas a los productores.

Se estima que las pérdidas embrionarias y abortos afectan a un $20 \%$ de las vacas preñadas, perjudicando la producción láctea (Kemmerling et al., 2009; Rojas et al., 2018). Chlamydophila abortus (C. abortus) es uno de los patógenos más frecuentemente relacionados a pérdidas reproductivas, y la vía de diseminación del patógeno está relacionada a descargas uterinas, placenta y secreciones reproductivas relacionadas al ternero abortado, contaminando durante meses las áreas de crianza (Entrican et al., 2001; Caro et al., 2009).

C. abortus es una bacteria gramnegativa intracelular obligada eucariota, y cuyo órgano diana es la placenta (Cheong et al., 2019; Everett et al., 2000; Longbottom y Coulter, 2003; Pospischil et al., 2010). Se desarrolla en un medio ambiente extremo, presentando un ciclo con dos formas: un cuerpo elemental (CE) infeccioso, metabólicamente inerte y un cuerpo reticulado (CR), metabólicamente activo que solo se encuentra dentro de las células (Caro et al., 2009; Orellano, 2016). Este patógeno pertenece al orden de los Clamidiales, familia Chlamidiaceae y presentan dos géneros, Chlamydia y Chlamidophila, con nueve especies en total (Longbottom y Coutler, 2003; Quinn et al., 2005).

C. abortus es un patógeno con amplia distribución mundial y es la mayor causa de abortos en vacas, ovejas, cabras, además de ser un agente zoonótico para el humano (Orellano, 2016; Cheong et al., 2019). Las infecciones ocurren mayormente en sistemas de crianza intensiva debido a la concentración de animales, sobre todo en periodos de parto (Karlsson et al., 2010; Bhandi et al., 2019). La transmisión ocurre principalmente a través de la ruta horizontal por ingestión o inhalación, pero también se reporta la transmisión venérea, dependiendo de la carga bacteriana en el semen (Cheong et al., 2019).

C. abortus normalmente ingresa a las tonsilas, para luego diseminarse por la sangre y linfa. En animales no gestantes permanece latente en los tejidos linfoides (Da Silva et al., 2006). El patógeno afecta la placenta (Pospischil et al., 2010), ocasionado pérdida de células epiteliales coriónicas, involucrando a los cotiledones y tejido adyacente, con necrosis focal, desencadenando el aborto en 
el último tercio de gestación (Albarracin, 2010) o el parto prematuro (Orellano, 2016). La respuesta inmune en el bovino es efectiva para eliminar el patógeno y proporciona protección a la reinfección por la misma cepa, pero tiende a disminuir de cuatro a seis meses de la infección (Pospischil et al., 2010). Existen varios reportes sobre la enfermedad en el continente; así, se encontraron prevalencias en bovinos de 4.78\% en Argentina y $1.42 \%$ en Brasil en bovinos. En Perú se reporta más del 20\% (202/ 938) de ovinos seropositivos en la SAIS Túpac Amaru (Silva et al., 2009; Sánchez et al., 2018; Rojas et al., 2018).

Para un óptimo diagnóstico, se debe tomar muestras de sangre, tejidos fetales y membranas placentarias), así como una adecuada historia clínica de los casos presentados (Stuen y Longbotton, 2011). La Organización Mundial de Sanidad Animal (OIE) plantea como prueba diagnóstica primaria más sensible a la técnica de ELISA (OIE, 2019); sin embargo, existe la reactividad antigénica cruzada entre $C$. abortus y $C$. pecorum (Vretou et al., 2007; Sachse et al., 2009). Ante esto, se considera a la prueba de ELISA como la más específica y de mayor eficacia diagnóstica para $C$. abortus (Longbottom y Coulter, 2003). Por todo ello, el estudio tuvo como objetivo evaluar la presencia de anticuerpos de C. abortus en el ganado bovino de crianza extensiva.

\section{Materiales Y MÉTOdos}

La investigación se realizó en los distritos Chumpi (3207 msnm), Coracora (3178 $\mathrm{msnm}$ ) y Pullo (2996 msnm) de la provincia de Parinacochas, ubicada al sur del departamento de Ayacucho, Perú, donde la crianza de ganado vivo se lleva a cabo bajo un sistema de tipo extensivo y bajo monta natural con un número de bovinos de forma muy irregular por ganadero, desde 10 a 200 animales aproximadamente.
El cálculo del tamaño de muestra se realizó según la fórmula de tamaño de muestra para poblaciones finitas (Wayne, 2012). La población bovina total era de 49 275, distribuidos en 27063 cabezas en Parinacochas, 13988 en Coracora, 7736 en Pullo y 5339 en Chumpi. Se consideró una prevalencia de 4.78\% reportada por Rojas et al. (2018) en Argentina, un nivel de confianza de $95 \%$ y un error de 5\%. El tamaño de muestra resultante fue de 62 bovinos, las cuales correspondieron a 12 para Chumpi, 32 para Coracora y 18 para Pullo. No obstante, el número de muestras se incrementó a 184 bovinos procedentes de 12 hatos ganaderos a fin de minimizar el error de muestreo y por disponibilidad de materiales.

La selección de muestras fue aleatoria. Se escogieron animales mayores de 4 meses, indistintamente del sexo de cada establecimiento. La sangre fue colectada por punción de la vena yugular en tubos al vacío de $10 \mathrm{ml}$. Las muestras fueron llevadas al laboratorio del Centro de Salud de la ciudad de Coracora, centrifugadas a $1600 \mathrm{~g}$ durante 5 minutos y el suero resultante fue congelado a $-20{ }^{\circ} \mathrm{C}$ hasta su análisis en el laboratorio de Microbiología y Microscopía Veterinaria de la Universidad Científica del Sur, Lima, para confirmar la presencia de anticuerpos de $C$. abortus mediante la técnica de ELISA indirecto con el kit comercial IDEXX Chlamydiosis Total Ab Test.

La seroprevalencia se expresó en porcentaje. Asimismo, se determinó la seroprevalencia corregida mediante la fórmula de Thrusfield (1990) tomando en cuenta la sensibilidad (99.7\%) y especificidad (100\%) del kit diagnóstico, con sus respectivos intervalos de confianza (IC) al 95\%. Además, se evaluó la asociación entre la presencia de anticuerpos contra $C$. abortus con la edad, sexo y procedencia de los bovinos mediante la prueba de Chi cuadrado. 


\section{Resultados}

El $40.34 \pm 7.06 \%(74 / 184)$ de los animales resultó positivo a anticuerpos contra C. abortus (Cuadro 1). A nivel de los distritos, Pullo presentó la mayor prevalencia $(56.05 \pm 7.15 \% ; p<0.05)$. De otra parte, no se encontró asociación significativa con las demás variables (sexo, categoría zootécnica y raza). En la variable estado reproductivo, si bien hubo una gran diferencia numérica, el pequeño número de vacas preñadas muestreadas no permitió definir la asociación con la prevalencia del patógeno.

El diagrama de caja y bigotes en la Figura 1 muestra la dispersión de los valores de las edades de los bovinos evaluados en el estudio. La mediana fue de 5 años, pudiéndose determinar una simetría de edades para ambos resultados. Simetría similar fue observada entre el número de partos y los resultados a la prueba diagnóstica, donde el rango de animales tanto negativos como positivos a la prueba varió entre 1 y 6 partos, con una mediana de tres partos.

\section{Discusión}

La prevalencia corregida de anticuerpos contra C. abortus fue de $40.34 \pm$ $7.06 \%(74 / 184)$, siendo posiblemente el primer reporte de esta enfermedad en el área de influencia del trabajo. Estudios a nivel mundial reportan tasas de prevalencia entre 4.78 y $71 \%$ de seropositividad (Wang et al., 2001; Rojas et al., 2018), mostrando que la enfermedad tiene un comportamiento muy variable, potencialmente influenciado por factores como la especie de rumiante, el número de animales por área en sistemas de crianza intensiva, el contacto con otros rumiantes domésticos o silvestres, la modalidad de infección (alimentos contaminados, ingestión de tejidos placentarios, inhalación de aerosoles) (Nietfeld, 2001; Wang et al., 2001; Ndengu et al., 2018; Rojas et al., 2018; Bhandi et al., 2019).

C. abortus es reconocida como el patógeno más común causante de abortos infecciosos y de pérdidas reproductivas en el ganado bovino, ovino y caprino (Longbottom

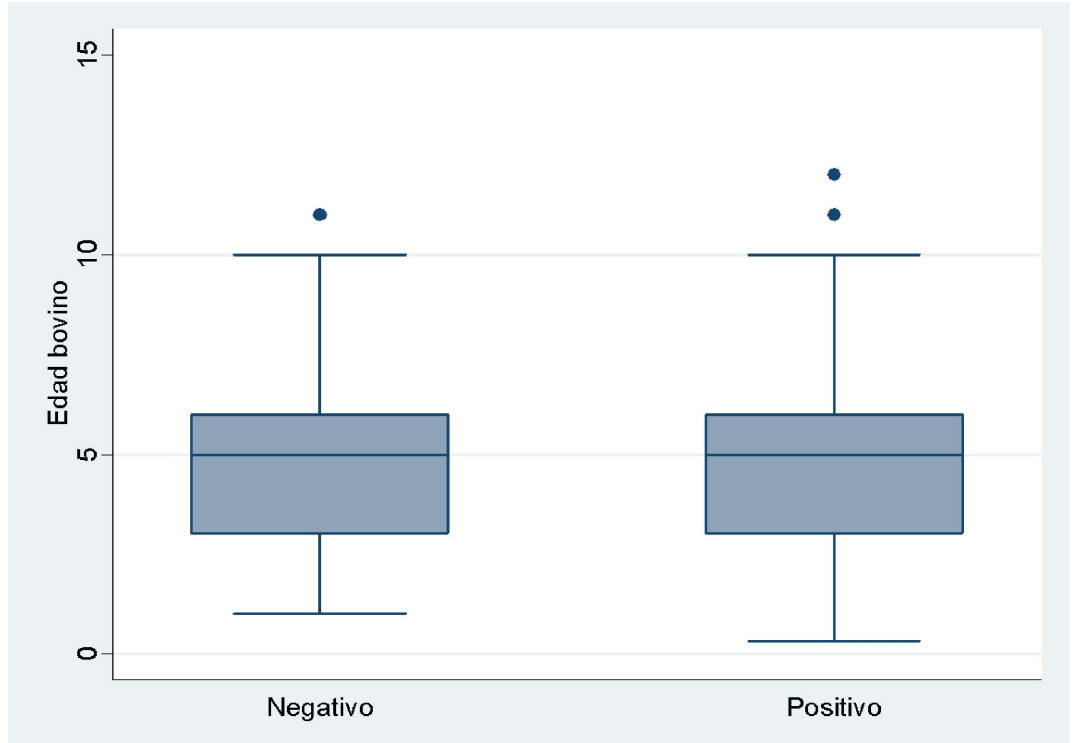

Figura 1. Diagrama de caja de bigotes de la edad de los bovinos evaluados según resultado a la prueba diagnóstica de Chlamydophilia abortus 
Cuadro 1. Seroprevalencia de Chlamydophila abortus $(\mathrm{n}=184)$ en bovinos de crianza extensiva de los distritos de Coracora, Chumpi, y Pullo de la provincia de Parinacochas, Ayacucho (2018)

\begin{tabular}{|c|c|c|c|c|c|}
\hline Variable $^{1}$ & & $\begin{array}{c}\text { Total } \\
\text { (n) }\end{array}$ & $\begin{array}{l}\text { Positivo } \\
\text { (n) }\end{array}$ & $\begin{array}{l}\text { Prevalencia }(\%) \\
\pm \text { IC }\end{array}$ & $\begin{array}{l}\text { Prevalencia } \\
\text { corregida }(\%) \\
\quad \pm \mathrm{IC}\end{array}$ \\
\hline \multirow[t]{2}{*}{ Sexo } & Macho & 20 & 7 & $35.00 \pm 6.87$ & $35.11 \pm 6.87$ \\
\hline & Hembra & 164 & 67 & $40.85 \pm 4.47$ & $40.97 \pm 7.08$ \\
\hline \multirow[t]{6}{*}{ Categoría } & Ternero & 1 & 1 & $100.00 \pm 4.32$ & $100.30 \pm 4.33$ \\
\hline & Vaquilla & 13 & 7 & $53.85 \pm 7.18$ & $54.01 \pm 7.18$ \\
\hline & Torete & 13 & 3 & $23.08 \pm 6.07$ & $23.15 \pm 6.07$ \\
\hline & Vaca & 144 & 57 & $39.58 \pm 7.04$ & $39.70 \pm 7.05$ \\
\hline & Toro & 6 & 3 & $50.00 \pm 7.20$ & $50.15 \pm 7.20$ \\
\hline & Vaquillona & 7 & 3 & $42.87 \pm 7.13$ & $42.99 \pm 7.13$ \\
\hline \multirow[t]{4}{*}{ Raza } & Criollo & 103 & 42 & $40.78 \pm 7.08$ & $40.90 \pm 7.08$ \\
\hline & Holstein & 4 & 2 & $50.00 \pm 7.20$ & $50.15 \pm 7.20$ \\
\hline & $\begin{array}{l}\text { Brown } \\
\text { Swiss }\end{array}$ & 44 & 22 & $50.00 \pm 7.20$ & $50.15 \pm 7.20$ \\
\hline & Simmental & 33 & 8 & $24.24 \pm 6.17$ & $24.31 \pm 6.18$ \\
\hline \multirow[t]{3}{*}{ Procedencia } & Chumpi & 33 & 8 & $24.24 \pm 6.17$ & $24.31 \pm 6.18$ \\
\hline & Coracora & 117 & 47 & $40.17 \pm 7.06$ & $40.29 \pm 7.06$ \\
\hline & Pullo & 34 & 19 & $55.88 \pm 7.15$ & $56.05 \pm 7.15$ \\
\hline \multirow{3}{*}{$\begin{array}{l}\text { Estado } \\
\text { reproductivo }\end{array}$} & Vacía & 140 & 54 & $38.57 \pm 7.01$ & $38.69 \pm 7.01$ \\
\hline & Preñada & 4 & 3 & $75.00 \pm 6.24$ & $75.23 \pm 6.22$ \\
\hline & Total & 184 & 74 & $40.22 \pm 7.06$ & $40.34 \pm 7.06$ \\
\hline
\end{tabular}

${ }^{1}$ Solo la variable procedencia presentó asociación significativa $(p<0.05)$ frente a anticuerpos de C. abortus

y Coulter, 2003; Edmondson et al., 2012; Sánchez et al., 2018). Solomon Bhandi et al. (2019) indicaron la relación de la seropositividad de $C$. abortus en cabras y el contagio con cabras de vida silvestre en Zimbabwe, en tanto que Ndengu et al. (2018) presentaron asociaciones similares entre bovinos y rumiantes de vida silvestre como búfalos $(47.7 \%)$ e impalas (43.8\%). El hacinamiento en el presente estudio no fue un fac- tor de importancia, toda vez que los animales se encontraban bajo condiciones de crianza extensiva, por lo que el contagio podría deberse a pastos contaminados y aerosoles de animales infectados (Kauffold et al., 2014). Sin embargo, a pesar de que la crianza es extensiva, los animales en la región de estudio son movilizados continuamente por callejones, puntos de toma de agua y áreas de descanso, que son compartidos por el gana- 
do de diferentes propietarios favoreciendo la transmisión de esta enfermedad.

La procedencia de los animales mostró una asociación significativa $(\mathrm{p}<0.05)$ con el diagnóstico positivo. La localidad de Pullo fue la más afectada, con una prevalencia corregida de $56.05 \% \pm 7.15 \%$ mientras la localidad de Coracora fue la menos afectada (40.29 $\pm 7.06 \%$ (Cuadro1). Esta asociación puede deberse a una mayor densidad animal por ganadero (Al-Qudah et al., 2004) y a las condiciones medio ambientales más adversas encontradas en el distrito de Pullo (Longbottom y Coulter, 2003).

\section{Conclusiones}

- La prevalencia corregida de anticuerpos contra Chlamydophila abortus en tres distritos de la provincia de Parinacochas (Ayacucho, Perú) fue de $40.34 \pm 7.06 \%$ (74/184).

- La variable de procedencia (distrito) presentó asociación significativa $(\mathrm{p}<0.05)$ con la seropositividad a la infección por C. abortus.

\section{Literatura Citada}

1. Albarracin F. 2010. Clamidiosis bovina. Tesis de Médico Veterinario Zootecnista. Ecuador: Univ. de Cuenca. $64 \mathrm{p}$.

2. Al-Qudah K, Sharif L, Raouf R, Hailat N. 2004. Seroprevalence of antibodies to Chlamydophila abortus in Awassi sheep and local goats in Jordan. Vet Med-Czech 49: 460-466. doi: 10.17221/ 5740-VETMED

3. Aronés M, Castillo L. 2003. Un breve perfil de Parinacochas. En: Huber L (ed). Ayacucho centralismo y descentralización. Perú: Instituto de Estudios Peruanos. p 291-292.
4. Bhandi S, Pfukenyi DM, Matope G, Murondoti A, Tivapasi M, Ndengu M, Scacchia M, et al. 2009. Brucellosis and chlamydiosis seroprevalence in goats at livestock-wildlife interface areas of Zimbabwe. Onderstepoort J Vet 86: e1e9. doi: 10.4102/ojvr.v86i1.1670

5. Caro MR, Buendía AJ, Del Rio L, Ortega N, Gallego MC, Cuello F, Navarro JA, et al. 2009. Chlamydophila abortus infection in the mouse: a useful model of the ovine disease. Vet Microbiol 135: 103-111. doi: 10.1016/j.vetmic.2008.09.029

6. Cheong HC, Lee CYQ, Cheok YY, Tan GMY, Looi CY, Wong WF. 2019. Chlamydiaceae: diseases in primary hosts and zoonosis. Microorganisms 7: 146. doi: 10.3390/microorganisms7050146

7. Da Silva F, De Freitas J, Muller E. 2006. Chlamydophila abortus em animais de produção. Cienc Rural 36: 342-348. doi: 10.1590/s013-84782006000100057

8. Edmondson M, Roberts J, Baird A. 2012. Teriogenología de ovinos y caprinos. En: Pugh D, Baird A (eds). Sheep and goat medicine. $2^{\circ}$ ed. Missouri: Elsiever. p 150-230.

9. Entrican G, Buxton D, Longbottom D. 2001. Chlamydial infection in sheep: immune control versus fetal pathology. J Roy Soc Med 94: 273-277. doi: 10.1177/ 014107680109400605

10. Everett KD. Chlamydia and Chlamydiales: more than meets the eye. Vet Microbiol 75: 109-126. doi: 10.1016/s0378-1135(00)00213-3

11. [INEI] Instituto Nacional de Estadística e Informática. 2012. IV Censo Nacional Agropecuario: Existencia de ganado, aves, otros animales y colmenas. Lima. [Internet]. Disponible en: http://censos.inei.gob.pe/Cenagro/ redatam/ 
12. Longbottom D, Coulter LJ. 2003. Animal chlamydioses and zoonotic implications. J Comp Pathol 128: 217-244. doi: 10.1053/jcpa.2002.0629

13. Karlsson A, Alenius S, Bjorkman $C$. 2010. Investigation of Chlamydiaceae in semen and cauda epididymidis and seroprevalence of Chlamydophila abortus in breeding bulls. Acta Vet Scand 52: 2. doi: 10.1186/1751-0147-52-2

14. Kauffold J, Wenhrend A, Sigmarsson H. 2014. Chlamydia and Chlamydophilia in bovine reproduction. Clin Theriogenology 6: 251-254.

15. Kemmerling K, Müller U, Mielenz M, Sauerwein H. 2009. Chlamydophila species in dairy farms: polymerase chain reaction prevalence, disease association, and risk factors identified in a crosssectional study in Western Germany. J Dairy Sci 92: 4347-4354. doi: 10.3168/ jds.2009-2051

16. Ndengu M, Matope G, Tivapasi M, Scacchi M, Bonfini B, Pfukenyi DM. 2018. Sero-prevalence of chlamydiosis in cattle and selected wildlife species at a wildlife/livestock interface area of Zimbabwe. Trop Anim Health Prod 50: 1107-1117. doi: 10.1007/s11250-018$1536-4$

17. Nietfeld JC. 2001. Chlamydial infections in small ruminants. Vet Clin NAmFood A 17: 301-314. doi: 10.1016/s07490720(15)30030-x

18. [OIE] Organización Mundial de Salud Animal. 2019. Manual de las pruebas de diagnóstico y de las vacunas para los animales terrestres [Internet]. Disponible en: https://www.oie.int/es/normas/manual-terrestre/acceso-en-linea/

19. Orellano R. 2016. Agentes infecciosos causales de aborto de presentación frecuente en bovinos. Tesis de Médico Veterinario Zootecnista. Tandil. Univ. Nacional del Centro de la Provincia de Buenos Aires. 108 p.
20. Pospischil A, Borel N, Andersen A. 2010. Clamidia. En: Carlton L, Prescott J, Songer G, Thoen C. Patogenia de las infecciones bacterianas en animales. Ames, Iowa, USA: Wiley-Blackwell. p. 575-587.

21. Quinn P, Markey B, Carter M. 2005. Microbiología y enfermedades infecciosas veterinarias, Acribia. $1002 \mathrm{p}$.

22. Rojas M, Fort M, Bettermann S. 2018. Detección de Chlamydia abortus en pérdidas reproductivas de bovinos en la provincia de La Pampa, Argentina. Rev Argent Microbiol 50: 269-274. doi: 10.1016/j.ram.2017.10.002

23. Rosemberg M. 2012. Retos y perspectivas de la ganadería bovina en el Perú. Lima. [Internet]. Disponible en: http:// www.veterinariadigital.com/articulos/laganaderia-bovina-en-peru

24. Sachse K, Vretou E, Livingstone M, Borel N, Pospischil A, Longbottom D. 2009. Recent developments in the laboratory diagnosis of chlamydial infections. Vet Microbiol 135: 2-21. doi: 10.1016/j.vetmic.2008.09.040

25. Sánchez L, Pantoja C, Villacaqui E, Morales-Cauti S. 2018. Seroprevalencia de anticuerpos contra Chlamydophila abortus en ovinos de SAIS Túpac Amaru del departamento de Junín, Perú. REDVET 19(6). [Internet]. Disponible en: http://www.veteri-naria.org/ revistas/redvet/n060618/061823.pdf

26. Silva Z, Spohr A, Lima B, Dias A, Muller E, Neto J, Turilli C. 2009. Prevalência de anticorpos antiChlamydophila spp em propriedades rurais com histórico de aborto bovino no estado do Paraná. Pesqui Vet Brasil 3: 29. doi: 10.1590/S0100-736X2009000300005

27. Stuen S, Longbottom D. 2011. Treatment and control of chlamydial and rickettsial infections in sheep and goats. Vet Clin N Am-Food A 27: 213-233. doi: 10.1016/j.cvfa.2010.10.017 
28. Thrusfield M. 1990. Epidemiología veterinaria. España: Ed Acribia. 42 p.

29. Vretou E, Radouani F, Psarrou E. 2007. Evaluation of two commercial assays for the detection of Chlamydophila abortus antibodies. Vet Microbiol 123: 153-161. doi: 10.1016/ j.vetmic.2007.02.023
30. Wang FI, Shieh H, Liao YK. 2001. Prevalence of Chlamydophila abortus infection in domesticated ruminants in Taiwan. J Vet Med Sci 63: 1215-1220. doi: 10.1292/jvms.63.1215

31. Wayne D. 2012. Bioestadística base para el análisis de las ciencias de la salud. $4^{\mathrm{a}}$ ed. México DF: Limusa. 750 p. 